\title{
TOPOLOGY CONTROL OF VANET BASED ON THE MULTI-RADIO IN VEHICLE AND TRAFFIC FLOW IN ROAD NETWORK
}

\author{
Yiming Tian, Yang Lu, Kèyù Zhü, Xiaojuan Zhu, Shuwei Qiu
}

Preliminary communication

For mobile internet network (Vehicular Ad Hoc Networks, VANET), resources allocation, transportation industry regulations and service business requirements, this paper builds a stable and efficient mobile internet topology and designs multi-radio nodes in vehicle, and then raises the virtual-force oriented ant colony optimization algorithm through primal approach topology to abstract road network. Based on these ideas, we design the process of networking and maintenance in sections. Finally, we verify the feasibility and effectiveness of topology control strategy of VANET based on the multiradio in vehicle and traffic flow in road network through the simulation experiment.

Keywords: road network; topology control; traffic flow; VANET

Reguluiranje topologije VANET-a zasnovano na multi-radiju u vozilu i protoku prometa na cestovnoj mreži

Prethodno priopćenje

Za pokretnu mrežu interneta (Vehicular Ad Hoc Networks, VANET), određivanje resursa, pravila industrije transporta i potreba održavanja, rad daje sigurnu i učinkovitu topologiju mobilnog interneta i dizajnira multi-radio čvorove u vozilu, a zatim izrađuje algoritam optimizacije kolonije mrava usmjeren virtualnoj sili kroz topologiju izvornog pristupa kako bi stvorio abstraktnu cestovnu mrežu. Na osnovu tih ideja, gradimo postupak umrežavanja i održavanja u pojedinim dijelovima. Konačno provjeravamo izvedivost i učinkovitost strategije kontrole topologije VANET-a zasnovanu na multi-radiju u vozilu i toku prometa na cestovnoj mreži kroz simulirani eksperiment.

Ključne riječi: cestovna mreža; kontrola topologije; tok prometa; VANET

\section{Introduction}

The topology control problem in VANET for complex road network originates from business needs, which mainly includes three reasons. One reason is that the business needs of mobile internet network include VANET, ad hoc network and complex network; the second reason refers to the requirements of resource distribution, especially the restriction of limited resources such as bandwidth and real-time performance, the collection, sharing and application of big data demand. The last reason is that the requirements of regulation and service in transportation industry, the operation management of road network need a reliable and effective network structure to carry safety information and traffic information.

There are many researches and deployments about intelligent transportation systems in the United States, Japan and the EU [1]. For example, the study of traffic condition via road network in order to analyse its geometry and topology characteristics $[2 \div 5]$. The research method includes graph theory and evolutionary dynamics $[6 \div 8]$, traffic flow efficiency of traffic network and network evolution $[9 \div 11]$. From the view of VANET, refs. $[12 \div 15]$ discuss the general characteristics of ad hoc network, the space and time distribution of the nodes, the characteristics of car movement and the spread of the wireless signal.

The distribution of topology degree in scale-free network follows the power-law distribution, a small number of nodes have high degrees, but most nodes are relatively small, the network system structure presents non-uniformity. The failure of the nodes with small degrees will not affect the entire network topology structure, and show strong robustness [16]. At present there are few studies of VANET from the perspective of scale-free networks. Guarantee of topology structure and protection of VANET performance are important aspects, especially for the network which needs connectivity, coverage, delay, interference, capacity and other properties. The research is roughly divided into two aspects: the active topology control and simulation analysis. The main idea is that if one node fails, one can design effective topology maintenance algorithm to selfheal topology. The passive topology control: the main idea is that if one node fails, one can protect effective topology structure through the node's neighbouring relations. Given that the degree distribution obeys powerlaw distribution, the scale-free network has anti-damage ability to random node failure, some researchers have the structure of a scale-free network to tolerate node failure [17]. On control strategy, this paper applies the main idea of the Ant Colony Optimization algorithm. It is first proposed by Italian scholar, as an innovative metaheuristic algorithm. M. Dorigo and T. Stutzle have important contribution to the application and development of Ant Colony Optimization algorithm. They wrote Ant Colony Optimization in 2004 [19] and introduced algorithm mechanism in detail.

At present, because of the limitation of the information collection equipment in highway traffic in China, it is difficult to monitor and discriminate the operation state of the entire road traffic. The limited traffic information cannot ensure the safety of traffic operation and precautionary analysis. On the basis of the abstract network topology structure, we construct a complex network topology for vehicular internet.

\section{Highway traffic network topology based on section flows}

\subsection{Traffic flow}

The highway road network is a complex network composed of many roads and intersections. The highway network has direction, either traffic flow. Therefore, when 
the network abstracts, we can abstract the highway network as a directed graph. If only considering the network topological structure regardless of the traffic flow direction, the road network could be regarded as an indirect graph.

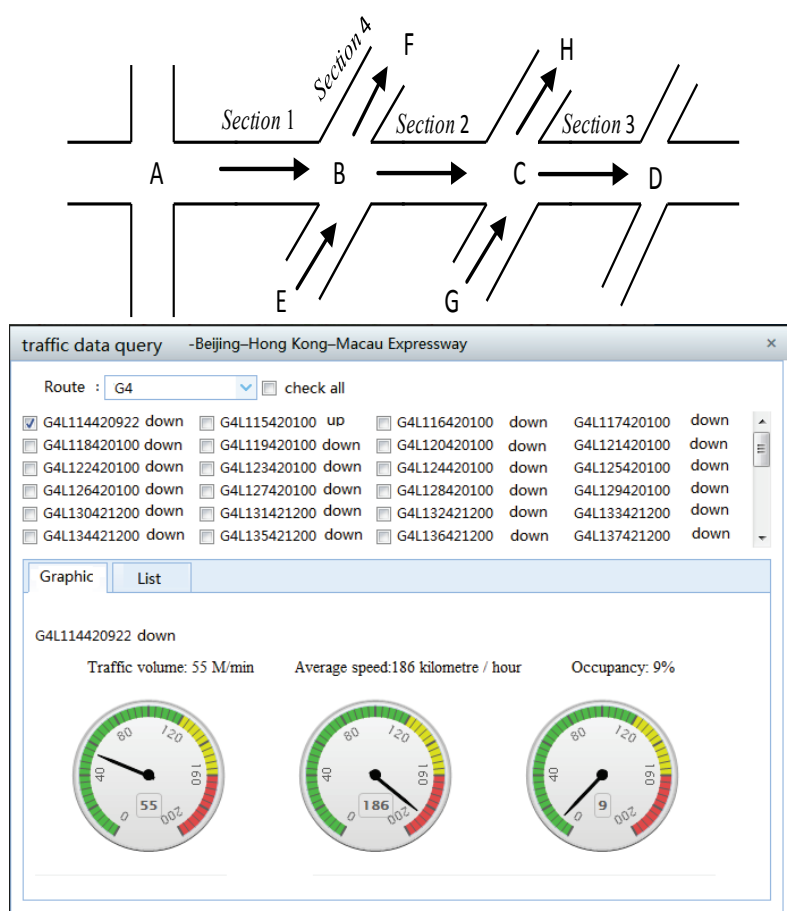

Figure 1 Propagation of traffic flow and Interface of query system

\subsection{Multi-radio nodes}

As shown in Fig. 2, multi-radio node function diagram mainly includes three parts: RF module, micro controller module and power module. We can increase storage, processing, mobile control module by business needs to extend the function of the network nodes.

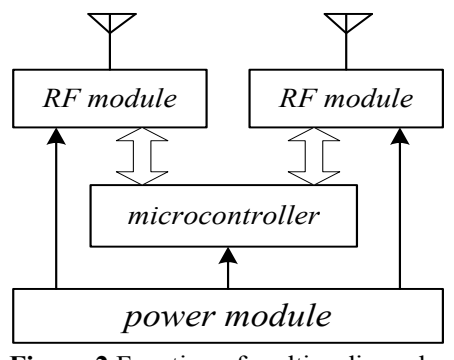

Figure 2 Function of multi-radio nodes

When the number of RF channels is less than that of available channels, one can switch in different channels by changing the frequency point. Of course, the delay from one channel switching to another is the cost of this mechanism. However, the frequent switch of RF will reduce the advantage of multi-channel communication. One can use a public channel to transfer control message in order to enhance spectrum resources efficiently and guarantee the connectivity of the network.

\subsection{Topology structure}

Due to the limited scope of monitoring, it is unable to cover entire road network. We propose that each section be taken as a subnet and the toll station road taken as node which is the sink node of each subnet. Fig. 3 is the map of Anhui highway and toll station deployment. Effective highway road network coverage can be realized according to each section of the traffic flow of subnet node deployment of traffic flow in each section, which will give data support for early warning and protection.

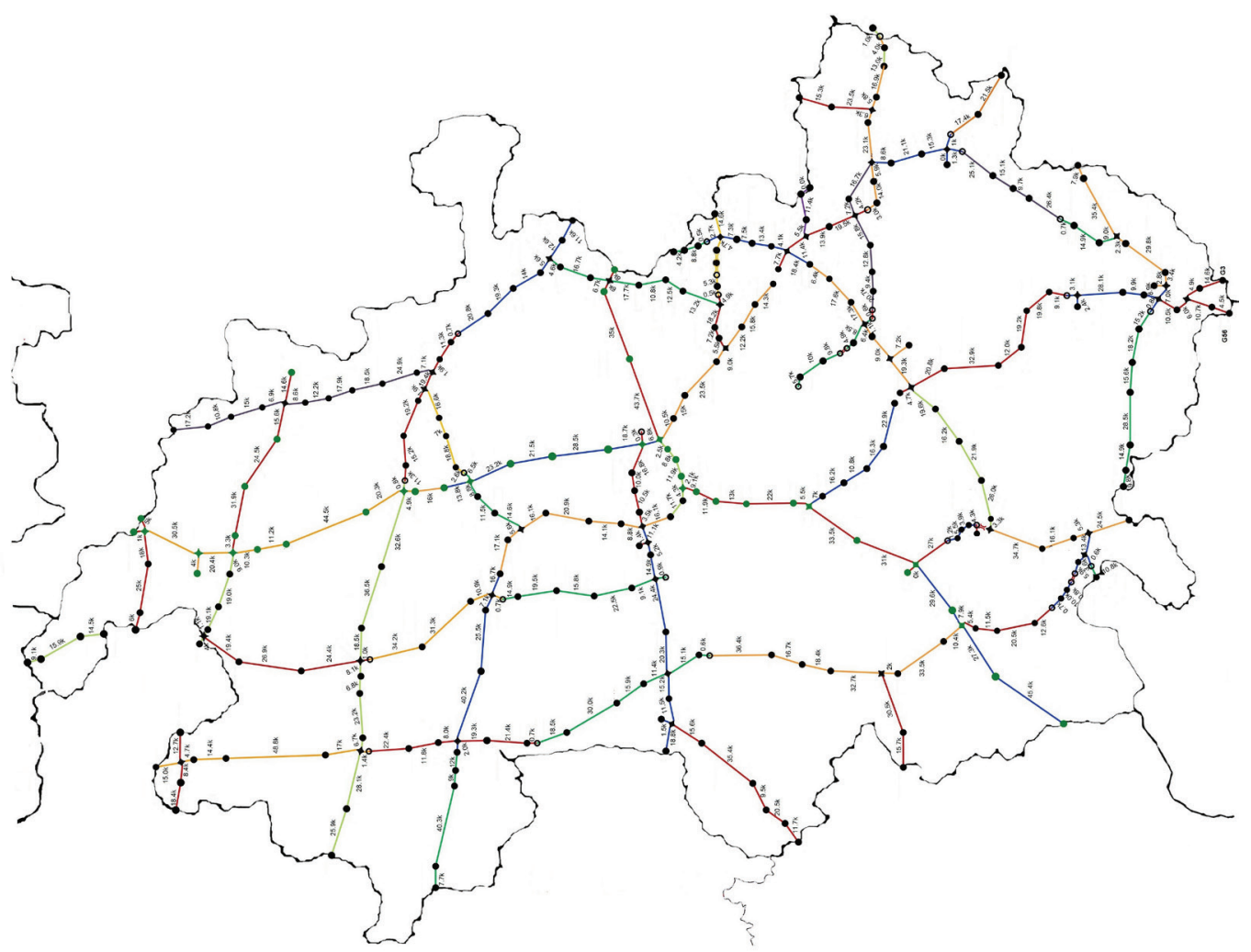

Figure 3 The toll station deployment in Anhui highway map 
There are three reasons considering the position and deployment of sink node. Firstly, sink node requires data processing ability and power demand. In reality, power supply system of toll station is perfect and equipped with high-performance node, so one can ignore the constraints of energy consumption and node living environment; secondly, network resources are relatively abundant and the connection between network nodes can use wire mode, which meets the demand of the structure of shortcuts; thirdly, shortcuts will increase the node degree. In the view of the entire road network, vital important node is the node of high degree value which can support the construction of scale-free networks. In addition, from the actual operation, both the design of wire shortcuts and the deployment of sink node are relatively easy to implement.

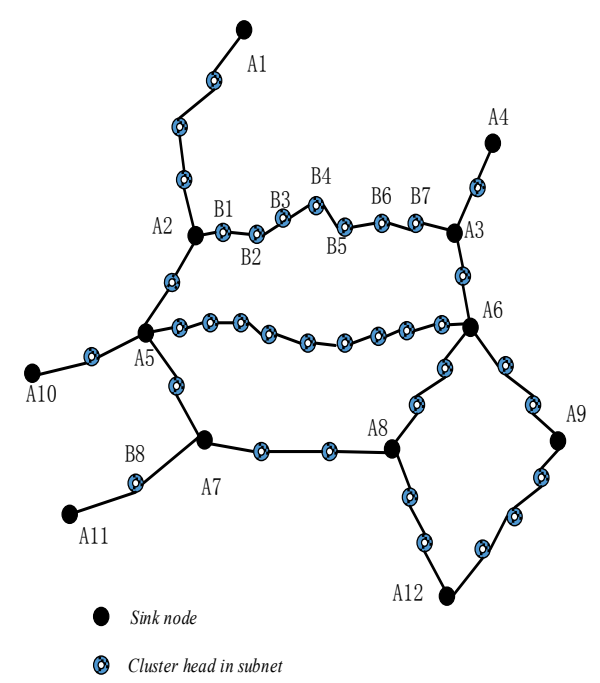

Figure 4 Highway topology based on section flow

Network can be abstracted as a set $\mathrm{G}=(\mathrm{V}, \mathrm{E})$ which is composed of a point set $\mathrm{V}$ and edge set $\mathrm{E}$. The collection of nodes is expressed in $\mathrm{V} 1=\{\mathrm{A} 1, \mathrm{~A} 2, \ldots, \mathrm{A} n\}$ and $\mathrm{V} 2=\{\mathrm{B} 1, \mathrm{~B} 2, \ldots, \mathrm{B} n\}$, and the collection of edges is expressed in $\mathrm{EA} 1=\{\mathrm{EA} 1, \mathrm{EA} 2, \ldots, \mathrm{EA} n\}$ and $\mathrm{EA} 2=$ $\{\mathrm{EB} 1, \mathrm{~EB} 2, \ldots, \mathrm{EB} n\}$. Highway network can be abstracted as the whole network $\mathrm{G} 1=(\mathrm{V} 1, \mathrm{E} 1)$ and local network $\mathrm{G} 2=(\mathrm{V} 2, \mathrm{E} 2) . \mathrm{G} 1$ is the overall distribution of the highway network, and G2 is chain network of localclustering in each section. Fig. 4 is the network topology abstracted from highway network.

Nodes in the network G1 are the sink nodes of each section (sink node A1, A2,.., A12, as shown). Each sink node can accept the traffic information in the corresponding section of the cluster head node, and the sink nodes distribute in highway toll station junction while the edge is the corresponding sections (e.g., sections A1-A2, A2-A3 and A5-A6 etc.).

Nodes in G2 network are cluster head nodes of each section's subnet (e.g., B1, B2,..., B8). In order to achieve efficient highway road network coverage to arrange nodes for traffic information collection, each section is divided into several clusters and each node is regarded as a cluster head node, which can cover a local area. Fig. 5 is the map of network topology, the cluster head node is dual RF node. $r_{1}$ is the communication radius of the first radio, $r_{2}$ is the second one, where $r_{2}>r_{1}$. Cluster head node uses the first radio to communicate with the mobile node in cluster. Meanwhile, one can upload traffic information to the sink node by using the second radio via multi-hop communication.

There are a large number of nodes and edges in the highway network topology. An adjacency matrix $\boldsymbol{A}_{i j}$ represents the structural information, and matrix element $a_{i j}$ represents the connection state of the node $i$ to $j$. If the node $i$ is connected with the node $j$, then $a_{i j}=1$; Otherwise, let $a_{i j}=0$.

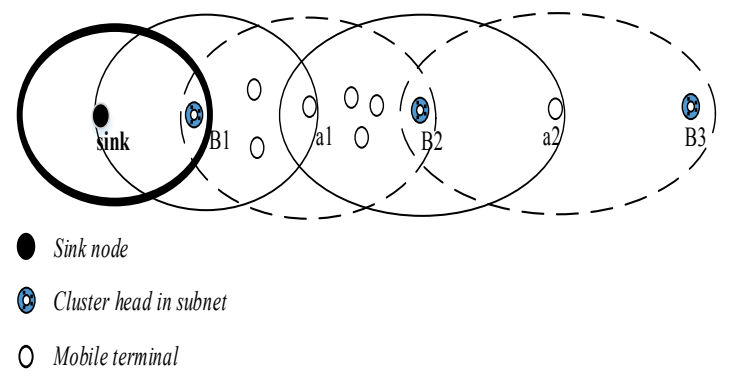

Figure 5 Clustering subnet topology

\section{Road network topology control optimization strategies 3.1 Related parameters}

Traffic flow parameters are used to describe the operation state characteristics of traffic flow. Usually one can describe the basic properties of traffic flow by observing the rule of variation of these parameters.

Definition 3.1: $Q=N / T$, the flow rate $Q$ refers to the number of vehicles $N$ on a road in a period of time $T$.

Definition 3.2: $\rho=N / L$, traffic density $\rho$ refers to the number of vehicles in a unit path length. $L$ refers to the length of the road. $N$ refers to the number of vehicles on this road.

Definition 3.3: $k=Q(T) / P(T), k$ is a ratio of the entering and exiting flow. $Q(T)$ is entering flow, $P(T)$ is exiting flow.

In general, $k$ can reflect the characteristics of the actual traffic capacity directly and accurately. If $k$ is bigger and the area also has a big traffic flow density, the traffic congestion is serious, and vice versa. Thus, one can employ the entering flow $Q(T)$ and the exiting flow $P(T)$ as criteria to evaluate the traffic capacity, especially the actual capacity of cross section of traffic accident

Definition 3.4: $\mu=2 m r / L, \mu$ is the coverage rate of highway node, $r$ is communication radius of the cluster head node, $m$ is the number of cluster head nodes layout in road, and $L$ is the total length of roads. Because of the chain structure of sections, in the case of no intersection among all monitoring nodes coverage area, we use the ratio of coverage area and total area to replace it.

\subsection{Virtual-force}

The purpose of virtual-force reference is to simulate the motion of two objects by driving force. A mathematical model was abstracted from the process, which is used to deploy mobile nodes in an ad hoc network. In this way, one can control the nodes within the global network system, and then meet the coverage control requirements. 
In wireless ad hoc network, virtual-force structure model is defined as follows:

$F=G_{0} \frac{r_{i}^{2} r_{j}^{2}}{d^{2}}$, where $r_{i}$ and $r_{j}$ are the communication radius, $d$ is the distance between two points, $G_{0}$ is a constant.

Like an electric field theory, the charged particles in electric field from the high potential point to the low potential. Virtual potential field also simulates this process: each node in the network is regarded as a virtual charge, meanwhile the goals and obstacles are considered as low potential points and high potential points. The force of potential field to charged network nodes can make the nodes move to the target and avoid obstacles in the moving process.

From a global network perspective, each node in the network has "charge." Depending on the different locations, in nodes exist the gravity and repulsion. Under the action of two virtual-forces, the node moves to the target area in other regions. Eventually, under the premise of the network connectivity, the nodes in the entire region reach the equilibrium state.

In order to achieve the target of adequate coverage in the target area and uniformity of the net coverage, the network coverage area may be maximized, which achieves the best coverage through control optimization. According to the above ideas, a network node has three neighbour nodes; virtual-forces received are expressed as follows:

$$
\vec{F}_{p}=\sum_{q=1, q \neq p}^{k} \vec{F}_{p q}+\vec{F}_{p R}+\vec{F}_{p A}
$$

\subsection{Niche ant colony algorithm}

Ant colony algorithm (ACA) is a meta-heuristic algorithm, which is proposed by Italian scholar Macro Dorigo. German scholar Thomas Stutzle also makes an important contribution to ACA and its application. They wrote a book Ant Colony Optimization [19] to demonstrate the mechanism of ACA. Ref [20] puts forward virtual-force oriented control link of ant colony optimization.

The main idea of niche model is described below. In the network scheme of vehicle with multi-radio, before starting the second RF (high power RF), the probability of non-communication state of the whole network is very high. If the network is un-connected, then take some nodes to start the second RF selectively. If the network is connected, in order to build scale-free network to shorten the shortest path of control link, we also need to start the second RF. This selection of un-connected network is intra-cluster selection of isolated island, where the cluster can be taken as niche, and the virtual-force oriented optimization strategy of ant colony proceeds simultaneously in this cluster. Meanwhile, the connected network launches multi-RF node as the cluster head directly, then creates a niche through setting threshold value for clustering. When multi-RF started, the model can obtain, post, update the whole network routing table for each cluster in radio frequency (RF) node through long-range connections which can serve as control link. Then update the pheromone and adjust distribution by virtual-force. Finally we achieve the optimization goal through cycle operation based on this strategy.

The above algorithm not only considers the generality of problem, but also the complexity of ant colony optimization algorithm, namely narrowing search range through niche to reduce algorithm complexity.

According to ACA, we have the following definitions:

One is the transition probability of ant colony.

Let the ant path selection probability is:

$$
p_{i j}^{k}=\left\{\begin{array}{cc}
\frac{\left[\tau_{i j}\right]^{\alpha}}{\sum_{l \in N_{i}^{k}\left[\tau_{i j}\right]^{\alpha}}} \quad j \in N_{i}^{k} \\
0 & \text { otherwise }
\end{array} .\right.
$$

$p_{i j}^{k}$ is the probability of ant $k$ in note $i$ may visit note $j ; \tau$ is the function of virtual-force; $\alpha$ represents the degree of information important; $N_{i}^{k}$ is the collection of ant $k$ in note $i$ come adjacent nodes directly.

The other is updating the ant pheromone

$\tau_{i j} \leftarrow(1-\rho) \tau_{i j}+\sum_{k=1}^{m} \Delta \tau_{i j}^{k}$

For ant $i$, we have:

$\Delta \tau_{i j}^{k}=\left\{\begin{array}{c}Q / C^{k}, \text { edge }(i, j) \text { in path } T^{k} \\ 0, \quad \text { otherwise }\end{array}\right.$

Here, $\rho$ is the evaporation rate of pheromone, which means the change rate of virtual force of node movement in each loop. $\Delta \tau_{i j}^{k}$ is the pheromone value released by ant $k$ from note $i$ to $j$. $Q$ represents the intensity of pheromone, it is the average of the sum of all the virtualforces on path. $C^{k}$ is the total expenditure of ant $k$ in all edges of path $T^{\mathrm{k}}$, it is the sum of all the virtual-forces on path. If there is no update, all ants will search in limited adjacent area of the last optimal path.

\subsection{Topology control optimization strategy}

At the start of topology construction, there are three principles for node's deployment:

(1) Cluster node is shortcut node, and it can transmit data directly to server in network management centre.

(2) Each section is arranged under traffic flow and traffic density. If the incoming flow and outgoing flow of the section is bigger than $k$, subnet node layout density is big, i.e. coverage rate $\mu$ is big, $\mu \infty k$.

(3) Section subnet node employs dual radio multichip communication. The first RF communication radius is $r 1$, the second RF communication radius is $r_{2}, r_{2}>2 r_{1}$. The cluster head node employs the first RF to communicate with the vehicles in clusters. Meanwhile, use the second 
RF to transmit traffic report with multichip communication to sink nodes.

Based on the above principles, we initialize the sink node position of highway network and relevant parameters, divide the road network into sections as well as subnet each section. The necessity of dividing subnets lies in the highway construction and operation in different areas being not the same. After dividing, we can analyse according to the specific operational aspect, and directly employ primal approach to cluster road network. It is the perspective of macro road network on the influence of traffic incidents, the perspective of section on a specific event, which also illustrates the necessity of clustering.

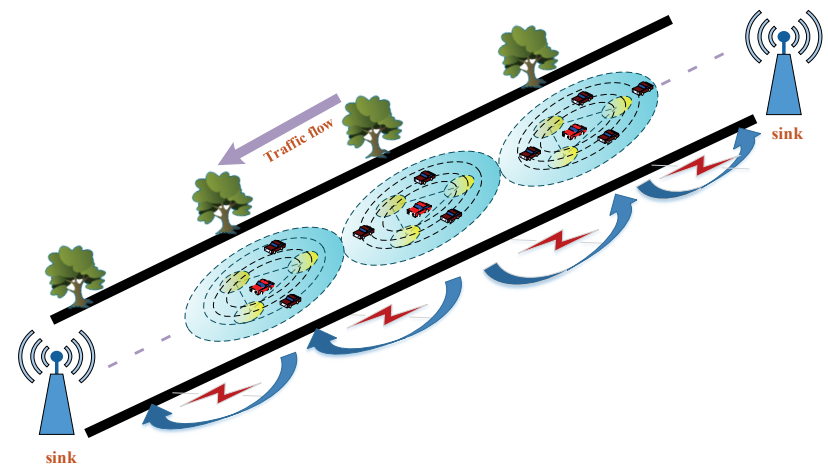

Figure 6 Section network structure

Mainly including:

Step 1: Create a highway electronic map and determine the coordinates of each section, then initialize highway road network sink node location (according to the electronic map, set the sink node in various highway intersections), according to the test data, initialize each road parameter (length, flow and density)

Step 2: Read traffic flow data of each road section, and calculate the ratio of incoming and outgoing flow.

Step 3: Calculate the coverage rate $\mu$ of cluster head node through the ratio of incoming and outgoing flow.

Step 4: Select road section and then choose cluster head node by cluster algorithm.

Step 5: If all road section are traversed, go to step 6; else, go to step 4.

Section 6: Test the road section parameter, the parameter changes then go to step 3; else, go to step 6 .

There is an advantage in setting toll for sink node, where one can obtain traffic section data through toll station in real time.

The core of the flow is cluster algorithm that can apply the network node to multi-radio frequency device.

Step 1: In the sink node coverage, in direction of the traffic flow, randomly select a mobile node $\mathrm{B} i$ as the cluster head node;

Step 2: Node $\mathrm{B} i$ starts double RF work mode;

Step 3: After node $\mathrm{B} i$ is selected as the cluster head node, start the first RF broadcasting $\mathrm{H}-\mathrm{Msg}$ message around, announce to be a cluster head node, receive the $\mathrm{H}-\mathrm{Msg}$ message node and give response, send a J-Msg request to the cluster head node to join the cluster message, after getting verification and agreement of cluster node $\mathrm{B} i$, the node officially joins cluster $\mathrm{B} i$, the cluster is identified as collection $\mathrm{CB} i=\{\mathrm{CB} i 1, \mathrm{CB} i 2, \ldots\}$;
Step 4: $\mathrm{B} i$ starts the second RF (the second RF communication radius is larger than the first $\mathrm{RF}$ communication radius), starts the first RF broadcasting $\mathrm{H}$ Msg message around, receives the H-Msg message node and gives response (the nodes in $C B i$ have been in cluster $\mathrm{B} i$ no response is given here), $\mathrm{B} i$ bases the position of the nodes, selects opposite direction of $\mathrm{B} i$ movement in all the response nodes identified as collection: $\mathrm{NB} i=$ $\{\mathrm{NB} i 1, \mathrm{NB} i 2, \ldots\}$.

Step 5: Use ant colony algorithm and the virtual-force to select $\mathrm{A} i$ adjacent cluster head nodes, A $i$ as a starting point, arrange $N$ ants, use the virtual-force to update the pheromone of ant, and select adjacent cluster head nodes in the collection NBi.

Step 6: Find adjacent cluster nodes, $i=i+1$, turn to Step 2, or to Step 7;

Step 7: Increase transmitted power of the second RF, turn to Step 4.

\section{Simulation analysis}

Before we describe the simulation work, there are two points to be mentioned here: (1) In the actual road network operation, if the roads are closed because of bad weather or other reasons, there are no vehicles on the highway, the premise we discuss in the paper cannot work, or only sink node can work; (2) The topology control indicator, here we set it as the coverage rate, the definition of the coverage rate is based on the route and the cluster-head nodes, and if we consider Intra-cluster communication, or Intra-cluster role transformation (a cluster member to a cluster head), the coverage rate then is the minimum value of the road network coverage rate, that is, the actual coverage rate is larger than $\mu$ value defined in 3.4.

We take $\mu$ as index in simulation experiment and use the cycle of time parameter to adjust the speed (i.e., the vehicle will appear in the network according to the time interval node pushed to network environment).

Let: $L=10000 ; r_{1}=50 ; r_{2}=800$, wherein, $N$ increases from 1 to 500, $m$ increases from 1 to 100 , step can be adjusted; consider the value of $\mu$, initialize $\mu=0,5$.

\section{Case one:}

We assume that the vehicle randomly appears on the road, and we do not take control strategy to build and maintain its appearance, the coverage rate is no rules and not high. If the time constraint is in a 580 cycle, since all must be in the road network (that is to say, all the vehicles are in the sub cycle of the road network), then the network has got certain coverage range. As shown in Fig. 7.

\section{Case two:}

We assume that the vehicle randomly appears on the road, and we take control strategy to build and maintain its appearance. From the curve of coverage rate, there are obvious improvements when comparing to the case one, as shown in Fig. 8. This figure shows: the coverage rate is in the scope of 0,92 or round about, the jitter means that there exists time delay in the process of vehicles' appearance, this performance can stand for topology control results objectively and actually. 


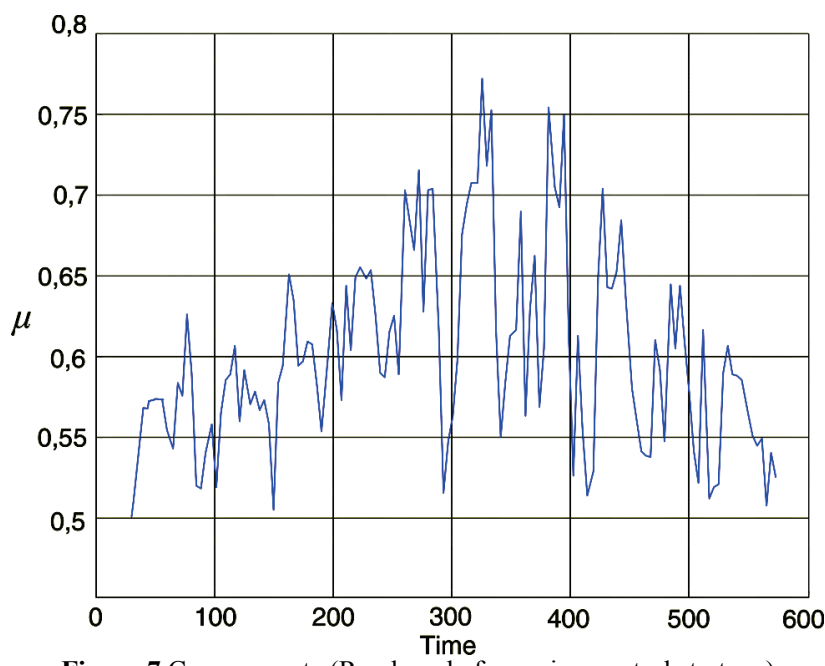

Figure 7 Coverage rate (Random, before using control strategy)

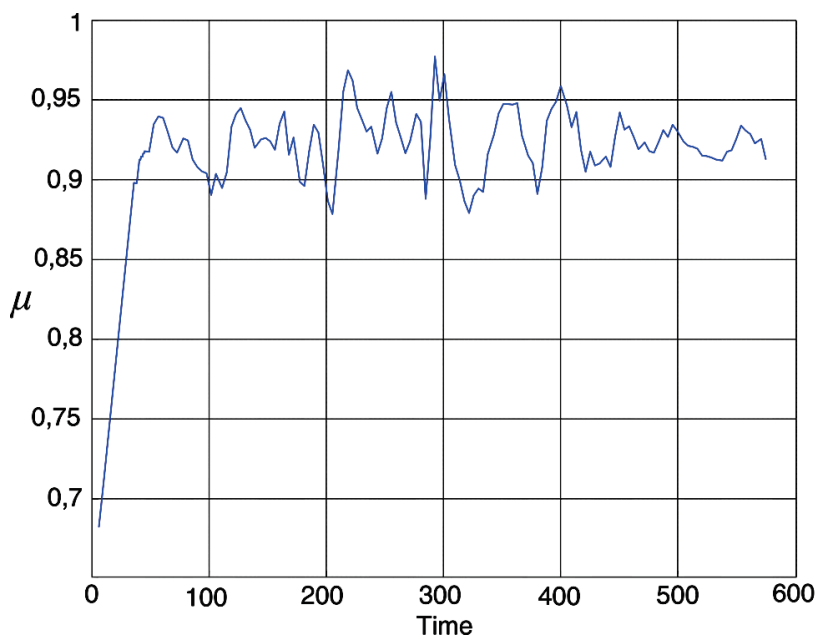

Figure 8 Coverage rate (Random, after using control strategy)

\section{Case three:}

We assume that the vehicle appears on the road at regular time interval, then the coverage rate can reach over $95 \%$, but there still exist jitters (see Fig. 9). When the vehicle appears randomly, the model requires optimization algorithm to execute the connecting process. It shows that the algorithm still has room for optimization. From the performance after 400 cycles, the network structure tends to be steady, consistent with the hypothesis.

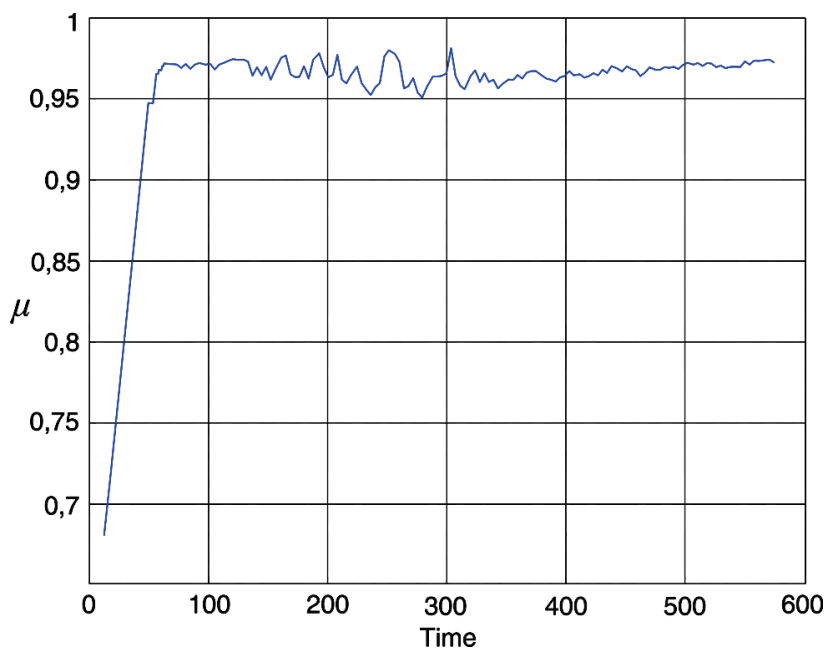

Figure 9 Coverage rate (Non-random, after using control strategy)
The above simulation results demonstrate the effectiveness of topology control based on section flow, and conform to road subnetwork business demand. If the sink nodes are based on cable and satellite link, the sink nodes are connected. From a global road network, the scale-free vehicle network topology is robust and maintainable. The highway network topology construction and maintenance control strategy based on the traffic flow can: provide two-tier topological structure (cluster nodes in subnetwork and sink nodes in road network) or three-tier topological structure (cluster, subnetwork and road network); as for the enhanced scalefree network invulnerability, keep the scale-free network to avoid key flaws, optimize the performance of scale-free network.

\section{Conclusions}

For the transportation industry regulation and service business needs, the paper here builds a stable and efficient complex road network, designs multi-radio nodes in vehicle and raises the virtual-force oriented ant colony optimization algorithm. Based on this algorithm, we design the road network and the maintenance process. Finally, we verify the feasibility and effectiveness of the vehicle with multi-radio network topology control strategy based on road network traffic flow through the simulation experiment.

\section{Acknowledgments}

We are very grateful to the editor and the anonymous referees for their valuable comments and suggestions. This research is supported by the national science foundation of China (NSFC) (No. 71501054, 71331002), national high technology research and development program (863 program) (No. 2011AA060406, 2012AA112308), the ministry of education university doctoral fund project (No. 20120111110001).

\section{References}

[1] An, S.; Lee, B. H.; Shin, D. R. A Survey of Intelligent Transportation Systems. // Proceedings of the Third International Conference on Computational Intelligence, Communication Systems and Networks. Bali, Indonesia, 2011, pp. 332-337. DOI: 10.1109/cicsyn.2011.76

[2] Liu, Kang; Duan, Yingying; Lu, Feng. Spatial Autocorrelation Analysis of Urban Road Traffic Based on Topological and Geometric Properties. // Journal of GeoInformation Science. 16, 3(2014), pp. 390-395.

[3] Cheng, T.; Haworth, J.; Wang, J. Spatio-temporal autocorrelation of road network data. // Journal of Geographical Systems. 14, 4(2012), pp. 389-413. (Chinese) DOI: 10.1007/s10109-011-0149-5

[4] Ding, Q. Y.; Wang, X. F.; Zhang, X. Y. et al. Forecasting traffic volume with space- time ARIMA model. // Advanced Materials Research. 156(2011), pp. 979-983.

[5] Zhou, Q.; Li, Z. Evaluation of Properties to Determine the Importance of Individual Roads for Map Generalization. // Advances in Cartography and GIScience. 5, 1(2011), pp. 459-475. DOI: 10.1007/978-3-642-19143-5_26

[6] Liu, Gang; Li, Yongshu; Yang, Jun; Zhang, Xiping. Autoselection Method of Road Networks Based on Evaluation 
of Node Importance for Dual Graph. // Acta Geodaetica et Cartographica Sinica. 43, 1(2014), pp. 97-104. (Chinese)

[7] Yang, B.; Luan, X.; Li, Q. Generating hierarchical strokes from urban street networks based on spatial pattern recognition. // International Journal of Geographical Information Science. 25, 12(2011), pp. 2025-2050. DOI: 10.1080/13658816.2011.570270

[8] Li, Shu-Bin; Wu, Jian-Jun; Gao, Zi-You; Lin, Yong; Fu, Bai-Bai. The analysis of traffic congestion and dynamic propagation properties based on complex network. // Acta physica sinica. 60, 5(2011), 050701. (Chinese)

[9] Wen-Xu, Wang; Bing-Hong, Wang; Bo, Hu; Gang, Yan; Qing, Ou. General Dynamics of Topology and Traffic on Weighted Technological Networks. // Physical Review Letter. 94, 18(2005), pp. 8702-8707.

[10] Nagumey, A.; Qiang, Q. Robustness of transportation networks subject to degradable links. // Europhysics Letters. 80, 6(2007), pp. 2007-2012.

[11] Qiang, Q.; Nagurney, A. A unified network performance measure with importance identification and the ranking of network components. // Optimization letters. 2, 1(2008), pp. 127-142. DOI: 10.1007/s11590-007-0049-2

[12] Prehofer, C.; Bettstetter, C. Self-organization in communication networks: Principles and design paradigms. // IEEE Communications Magazine. 43, 7(2005), pp. 78-85. DOI: 10.1109/MCOM.2005.1470824

[13] Tong, C.; Niu, J. W.; Qu, G. Z.; Long, X.; Gao, X. P. Complex networks properties analysis for mobile ad hoc networks. // IET Communications. 6, 4(2012), pp. 370-380. DOI: 10.1049/iet-com.2010.0651

[14] Sarshar, N.; Chowdhury, V. R. Multiple power-law structures in heterogeneous complex networks. // Phys. Rev. E. 72, 2(2005), pp. 6114-6124. DOl: 10.1103/PhysRevE.72.026114

[15] Sommer, P. Design and Analysis of Realistic Mobility Models for Wireless Mesh Networks. Switzerland, Swiss Federal Institute of Technology, 2007.

[16] Crucitti, P.; Latora, V.; Marchiori, M. et al. Error and attack tolerance of complex networks. // Physica A: Statistical Mechanics and its Applications. 340, 1-3(2004), pp. 388394.

[17] Younis, M.; Senturk, I. F.; Akkaya, K.; Lee, S.; Senel, F. Topology management techniques for tolerating node failures in wireless sensor networks: a survey. // Computer Networks. 58, (2014), pp. 254-283. DOl: 10.1016/j.comnet.2013.08.021

[18] Nicolau, M.; Schoenauer, M. On the evolution of scale-free topologies with a gene regulatory network model. // BioSystems. 10.1016/j.biosystems.2009.06.006

[19] Dorigo, M.; Stutzle, T. Ant Colony Optimization. / London, England: The MIT Press, 2014.

[20] Yiming, T.; Yang, L.; Fangzhen, G.; Qiang, W. Implementation Strategy of Global Control Link Based on Virtual-Force Directed Ant Colony Optimization in Wireless Sensor Networks. // Journal of computer research and development. 47(s), (2010), pp. 26-30. (Chinese)

\section{Authors' addresses}

\section{Yiming Tian}

Corresponding author

School of Computer \& Information,

Hefei University of Technology,

Anhui Transport Interconnection Management Center,

Hefei, 2300019, China

E-mail: lionbenz@163.com

Yang Lu,

School of Computer \& Information,

Hefei University of Technology, Hefei, 230009, China E-mail: Luyang.hf@126.com

\section{Kèyù Zhü}

Corresponding author

School of Management,

Hefei University of Technology, Hefei, 230009, China E-mail: cauchyandy@163.com

\section{Xiaojuan Zhu}

School of Computer \& Information,

Hefei University of Technology, Hefei, 230009, China E-mail: xjzhu@aust.edu.cn

\section{Shuwei Qiu}

School of Computer \& Information,

Hefei University of Technology, Hefei, 230009, China E-mail: qiusw@aiai.edu.cn 